\title{
The Intersection Complex as a Weight Truncation and an Application to Shimura Varieties
}

\section{Citation}

Morel, Sophie. 2011. The intersection complex as a weight truncation and an application to Shimura varieties. In ICM 2010: Proceedings of the International Congress of Mathematicians, Hyderabad, India, 19-27 August 2010, ed. R. Bhatia and A. Pal. Singapore, Hackensack, NJ: World Scientific Publishing Company.

\section{Permanent link}

http://nrs.harvard.edu/urn-3:HUL.InstRepos:4817365

\section{Terms of Use}

This article was downloaded from Harvard University's DASH repository, and is made available under the terms and conditions applicable to Open Access Policy Articles, as set forth at http:// nrs.harvard.edu/urn-3:HUL.InstRepos:dash.current.terms-of-use\#OAP

\section{Share Your Story}

The Harvard community has made this article openly available.

Please share how this access benefits you. Submit a story.

Accessibility 


\title{
The intersection complex as a weight trun- cation and an application to Shimura vari- eties
}

\author{
Sophie Morel *
}

\begin{abstract}
The purpose of this talk is to present an (apparently) new way to look at the intersection complex of a singular variety over a finite field, or, more generally, at the intermediate extension functor on pure perverse sheaves, and an application of this to the cohomology of noncompact Shimura varieties.
\end{abstract}

Mathematics Subject Classification (2000). Primary 11F75; Secondary 11G18, $14 \mathrm{~F} 20$.

Keywords. Shimura varieties, intersection cohomology, Frobenius weights

\section{Shimura varieties}

1.1. The complex points. In their simplest form, Shimura varieties are just locally symmetric varieties associated to certain connected reductive groups over $\mathbb{Q}$. So let $\mathbf{G}$ be a connected reductive group over $\mathbb{Q}$ satisfying the conditions in 1.5 of Deligne's article [17]. To be precise, we are actually fixing $\mathbf{G}$ and a morphism $h: \mathbb{C}^{\times} \longrightarrow \mathbf{G}(\mathbb{R})$ that is algebraic over $\mathbb{R}$. Let us just remark here that these conditions are quite restrictive. For example, they exclude the group $\mathbf{G} \mathbf{L}_{n}$ as soon as $n \geq 3$. The groups $\mathbf{G}$ that we want to think about are, for example, the group $\mathbf{G S p}_{2 n}$ (the general symplectic group of a symplectic space of dimension $2 n$ over $\mathbb{Q}$ ) or the general unitary group of a hermitian space over a quadratic imaginary extension of $\mathbb{Q}$. The conditions on $\mathbf{G}$ ensure that the symmetric space $\mathcal{X}$ of $\mathbf{G}(\mathbb{R})$ is a hermitian symmetric domain; so $\mathcal{X}$ has a canonical complex structure. Remember that $\mathcal{X}=\mathbf{G}(\mathbb{R}) / \mathrm{K}_{\infty}^{\prime}$, where $\mathrm{K}_{\infty}^{\prime}$ is the centralizer in $\mathbf{G}(\mathbb{R})$ of $h\left(\mathbb{C}^{\times}\right)$. In the examples we consider, $\mathrm{K}_{\infty}^{\prime}$ is the product of a maximal compact subgroup $\mathrm{K}_{\infty}$ of $\mathbf{G}(\mathbb{R})$ and of $A_{\infty}:=\mathbf{A}(\mathbb{R})^{0}$, where $\mathbf{A}$ is the maximal $\mathbb{Q}$-split torus of the center of G. (To avoid technicalities, many authors assume that the maximal $\mathbb{R}$-split torus in the center of $\mathbf{G}$ is also $\mathbb{Q}$-split. We will do so too.)

\footnotetext{
* This text was written while I was working as a Professor at the Harvard mathematics department and supported by the Clay Mathematics Institute as a Clay Research Fellow. I would like to thank the referee for their useful comments about the first version of this paper.
} 
The locally symmetric spaces associated to $\mathbf{G}$ are the quotients $\Gamma \backslash \mathcal{X}$, where $\Gamma$ is an arithmetic subgroup of $\mathbf{G}(\mathbb{Q})$, that is, a subgroup of $\mathbf{G}(\mathbb{Q})$ such that, for some (or any) $\mathbb{Z}$-structure on $\mathbf{G}, \Gamma \cap \mathbf{G}(\mathbb{Z})$ is of finite index in $\Gamma$ and in $\mathbf{G}(\mathbb{Z})$. If $\Gamma$ is small enough (for example, if it is torsion-free), then $\Gamma \backslash \mathcal{X}$ is a smooth complex analytic variety. In fact, by the work of Baily and Borel ([4]), it is even a quasi-projective algebraic variety.

In this text, we prefer to use the adelic point of view, as it leads to somewhat simpler statements. So let $\mathrm{K}$ be a compact open subgroup of $\mathbf{G}\left(\mathbb{A}_{f}\right)$, where $\mathbb{A}_{f}=$ $\widehat{\mathbb{Z}} \otimes_{\mathbb{Z}} \mathbb{Q}$ is the ring of finite adeles of $\mathbb{Q}$. This means that $K$ is a subgroup of $\mathbf{G}\left(\mathbb{A}_{f}\right)$ such that, for some (or any) $\mathbb{Z}$-structure on $\mathbf{G}, K \cap \mathbf{G}(\widehat{\mathbb{Z}})$ is of finite index in $K$ and in $\mathbf{G}(\widehat{\mathbb{Z}})$. Set

$$
S^{\mathrm{K}}(\mathbb{C})=\mathbf{G}(\mathbb{Q}) \backslash\left(\mathcal{X} \times \mathbf{G}\left(\mathbb{A}_{f}\right) / \mathrm{K}\right),
$$

where $\mathbf{G}(\mathbb{Q})$ acts on $\mathcal{X} \times \mathbf{G}\left(\mathbb{A}_{f}\right) / \mathrm{K}$ by the formula $(\gamma,(x, g \mathrm{~K})) \longmapsto(\gamma \cdot x, \gamma g \mathrm{~K})$.

This space $S^{\mathrm{K}}(\mathbb{C})$ is related to the previous quotients $\Gamma \backslash \mathcal{X}$ in the following way. By the strong approximation theorem, $\mathbf{G}(\mathbb{Q}) \backslash \mathbf{G}\left(\mathbb{A}_{f}\right) / \mathrm{K}$ is finite. Let $\left(g_{i}\right)_{i \in I}$ be a finite family in $\mathbf{G}\left(\mathbb{A}_{f}\right)$ such that $\mathbf{G}\left(\mathbb{A}_{f}\right)=\coprod_{i \in I} \mathbf{G}(\mathbb{Q}) g_{i} \mathrm{~K}$. For every $i \in I$, set $\Gamma_{i}=\mathbf{G}(\mathbb{Q}) \cap g_{i} \mathrm{~K} g_{i}^{-1}$. Then the $\Gamma_{i}$ are arithmetic subgroups of $\mathbf{G}(\mathbb{Q})$, and

$$
S^{\mathrm{K}}(\mathbb{C})=\coprod_{i \in I} \Gamma_{i} \backslash \mathcal{X}
$$

In particular, we see that, if $\mathrm{K}$ is small enough, then $S^{\mathrm{K}}(\mathbb{C})$ is the set of complex points of a smooth quasi-projective complex algebraic variety, that we will denote by $S^{\mathrm{K}}$. These are the Shimura varieties associated to $\mathbf{G}$ and $h: C^{\times} \longrightarrow \mathbf{G}(\mathbb{R})$ (over $\mathbb{C}$ ). From now on, we will assume always that the group $\mathrm{K}$ is small enough.

Remark 1. If $\mathbf{G}=\mathbf{G L}_{2}$, then $S^{\mathrm{K}}$ is a modular curve, or rather, a finite disjoint union of modular curves; it parametrizes elliptic curves with a certain level structure (depending on $\mathrm{K}$ ). Higher-dimensional generalizations of this are the Shimura varieties for the symplectic groups $\mathbf{G}=\mathbf{G S}_{2 n}$; they are called the Siegel modular varieties, and parametrize principally polarized abelian varieties with a level structure (depending on K). Some other Shimura varieties have been given a name. For example, if $\mathbf{G}$ is the general unitary group of a 3-dimensional hermitian vector space $V$ over an imaginary quadratic extension of $\mathbb{Q}$ such that $V$ has signature $(2,1)$ at infinity, then $S^{\mathrm{K}}$ is called a Picard modular surface.

1.2. The projective system and Hecke operators. If $K^{\prime} \subset K$ are two open compact subgroups of $\mathbf{G}\left(\mathbb{A}_{f}\right)$, then there is an obvious projection $S^{\mathrm{K}^{\prime}}(\mathbb{C}) \longrightarrow S^{\mathrm{K}}(\mathbb{C})$, and it defines a finite étale morphism $S^{\mathrm{K}^{\prime}} \longrightarrow S^{\mathrm{K}}$; if $\mathrm{K}^{\prime}$ is normal in $K$, then this morphism is Galois, with Galois group $K / K^{\prime}$. So we can see the Shimura varieties $S^{\mathrm{K}}$ as a projective system $\left(S^{\mathrm{K}}\right)_{\mathrm{K} \subset \mathbf{G}\left(\mathbb{A}_{f}\right)}$ indexed by (small enough) open compact subgroups of $\mathbf{G}\left(\mathbb{A}_{f}\right)$, and admitting a right continuous action of $\mathbf{G}\left(\mathbb{A}_{f}\right)$.

More generally, if $\mathrm{K}^{\prime}, \mathrm{K}$ are two open compact subgroups of $\mathbf{G}\left(\mathbb{A}_{f}\right)$ and $g \in$ $\mathbf{G}\left(\mathbb{A}_{f}\right)$, then we get a correspondence $\left[\mathrm{K}^{\prime} g \mathrm{~K}\right]: S^{\mathrm{K} \cap g^{-1} \mathrm{~K}^{\prime} g} \longrightarrow S^{\mathrm{K}} \times S^{\mathrm{K}^{\prime}}$ in the 
following way. The first map is the obvious projection $S^{\mathrm{K} \cap g^{-1} \mathrm{~K}^{\prime} g} \longrightarrow S^{\mathrm{K}}$, and the second map is the composition of the obvious projection $S^{\mathrm{K}^{-1} g^{-1} \mathrm{~K}^{\prime} g} \longrightarrow S^{g^{-1} \mathrm{~K}^{\prime} g}$

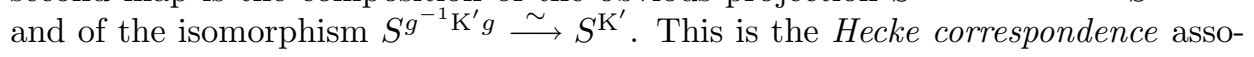
ciated to $g$ (and $\left.\mathrm{K}, \mathrm{K}^{\prime}\right)$.

Let $\mathrm{H}^{*}$ be a cohomology theory with coefficients in a ring $A$ that has good fonctoriality properties (for example, Betti cohomology with coefficients in $A$ ) and $\mathrm{K}$ be an open compact subgroup of $\mathbf{G}\left(\mathbb{A}_{f}\right)$. Then the Hecke correspondences define an action of the Hecke algebra at level $\mathrm{K}, \mathcal{H}_{\mathrm{K}}(A):=C\left(\mathrm{~K} \backslash \mathbf{G}\left(\mathbb{A}_{f}\right) / \mathrm{K}, A\right.$ ) (of bi$\mathrm{K}$-invariant functions from $\mathbf{G}\left(\mathbb{A}_{f}\right)$ to $A$, with the algebra structure given by the convolution product), on the cohomology $\mathrm{H}^{*}\left(S^{\mathrm{K}}\right)$. For every $g \in \mathbf{G}\left(\mathbb{A}_{f}\right)$, we make $\mathbb{1}_{\mathrm{K} g \mathrm{~K}} \in \mathcal{H}_{\mathrm{K}}(A)$ act by the correspondence $\left[\mathrm{K} g^{-1} \mathrm{~K}\right]$.

Let $\mathcal{H}(A)=\bigcup_{\mathrm{K}} \mathcal{H}_{\mathrm{K}}(A)=C_{c}^{\infty}\left(\mathbf{G}\left(\mathbb{A}_{f}\right), A\right)$ (the algebra of locally constant functions $\mathbf{G}\left(\mathbb{A}_{f}\right) \longrightarrow A$ with compact support) be the full Hecke algebra, still with the product given by convolution. Then we get an action of $\mathcal{H}(A)$ on the limit $\underset{\overrightarrow{\mathrm{K}}}{\lim } \mathrm{H}^{*}\left(S^{\mathrm{K}}\right)$. So the $A$-module $\underset{\mathrm{K}}{\lim _{\overrightarrow{\mathrm{C}}}} \mathrm{H}^{*}\left(S^{\mathrm{K}}\right)$ admits an action of the group $\mathbf{G}\left(\mathbb{A}_{f}\right)$.

1.3. Canonical models. Another feature of Shimura varieties is that they have so-called canonical models. That is, they are canonically defined over a number field $E$, called the reflex field, that depends only on $\mathbf{G}$ and the morphism $h: \mathbb{C}^{\times} \longrightarrow \mathbf{G}(\mathbb{R})$ (in particular, it does not depend on the open compact subgroup $\mathrm{K}$ of $\mathbf{G}\left(\mathbb{A}_{f}\right)$ ). We will use the same notation $S^{\mathrm{K}}$ for the model over $E$. Here "canonically" means in particular that the action of $\mathbf{G}\left(\mathbb{A}_{f}\right)$ on the projective system $\left(S^{\mathrm{K}}\right)_{\mathrm{K}}$ is defined over $E$. The theory of canonical models was begun by Shimura, and then continued by Deligne, Borovoi, Milne and Moonen (cf [17], [18], [13], [46], [47], [51]).

So, if the cohomology theory $\mathrm{H}^{*}$ happens to make sense for varieties over $E$ (for example, it could be $\ell$-adic étale cohomology, with or without supports), then the limit $\underset{\mathrm{K}}{\mathrm{K}} \mathrm{H}^{*}\left(S^{\mathrm{K}}\right)$ admits commuting actions of $\mathbf{G}\left(\mathbb{A}_{f}\right)$ and of $\operatorname{Gal}(\bar{E} / E)$. Another way to look at this is to say that the cohomology group at finite level, $\mathrm{H}^{*}\left(S^{\mathrm{K}}\right)$, admits commuting actions of $\mathcal{H}_{\mathrm{K}}(A)$ and of $\operatorname{Gal}(\bar{E} / E)$.

The goal is now to understand the decomposition of those cohomology groups as representations of $\mathbf{G}\left(\mathbb{A}_{f}\right) \times \operatorname{Gal}(\bar{E} / E)$ (or of $\mathcal{H}_{\mathrm{K}}(A) \times \operatorname{Gal}(\bar{E} / E)$ ).

1.4. Compactifications and the choice of cohomology theory. If the Shimura varieties $S^{\mathrm{K}}$ are projective, which happens if and only if the group $\mathbf{G}$ is anisotropic over $\mathbb{Q}$, then the most natural choice of cohomology theory is simply the étale cohomology of $S^{\mathrm{K}}$. There is still the question of the coefficient group $A$. While the study of cohomology with torsion or integral coefficients is also interesting, very little is known about it at this point, so we will restrict ourselves to the case $A=\overline{\mathbb{Q}}_{\ell}$, where $\ell$ is some prime number.

Things get a little more complicated when the $S^{\mathrm{K}}$ are not projective, and this is the case we are most interested in here. We can still use ordinary étale cohomology or étale cohomology with compact support, but it becomes much harder to study (among other things, because we do not have Poincaré duality or the fact that 
the cohomology is pure - in Deligne's sense - any more). Nonetheless, it is still an interesting problem.

Another solution is to use a cohomology theory on a compactification of $S^{\mathrm{K}}$. The author of this article knows of two compactifications of $S^{\mathrm{K}}$ as an algebraic variety over $E$ (there are many, many compactifications of $S^{\mathrm{K}}(\mathbb{C})$ as a topological space, see for example the book [11] of Borel and $\mathrm{Ji}$ ) :

(1) The toroidal compactifications. They are a family of compactifications of $S^{\mathrm{K}}$, depending on some combinatorial data (that depends on $\mathrm{K}$ ); they can be chosen to be very nice (i.e. projective smooth and with a boundary that is a divisor with normal crossings).

(2) The Baily-Borel (or minimal Satake, or Satake-Baily-Borel) compactification $\bar{S}^{\mathrm{K}}$. It is a canonical compactification of $S^{\mathrm{K}}$, and is a projective normal variety over $E$, but it is very singular in general.

See the book [3] by Ash, Mumford, Rapoport and Tai for the construction of the toroidal compactifications over $\mathbb{C}$, the article [4] of Baily and Borel for the construction of the Baily-Borel compactification over $\mathbb{C}$, and Pink's dissertation [55] for the models over $E$ of the compactifications.

The problem of using a cohomology theory on a toroidal compactification is that the toroidal compactifications are not canonical, so it is not easy to make the Hecke operators act on their cohomology. On the other hand, while the Baily-Borel compactification is canonical (so the Hecke operators extend to it), it is singular, so its cohomology does not behave well in general. One solution is to use the intersection cohomology (or homology) of the Baily-Borel compactification. In the next section, we say a little more about intersection homology, and explain why it might be a good choice.

\section{Intersection homology and $L^{2}$ cohomology}

2.1. Intersection homology. Intersection homology was invented by Goresky and MacPherson to study the topology of singular spaces (cf [24], [25]). Let $X$ be a complex algebraic (or analytic) variety of pure dimension $n$, possibly singular. Then the singular homology groups of $X$ (say with coefficients in $\mathbb{Q}$ ) do not satisfy Poincaré duality if $X$ is not smooth. To fix this, Goresky and MacPherson modify the definition of singular homology in the following way. First, note that $X$ admits a Whitney stratification, that is, a locally finite decomposition into disjoint connected smooth subvarieties $\left(S_{i}\right)_{i \in I}$ satisfying the Whitney condition (cf [24] 5.3). For every $i \in I$, let $c_{i}=n-\operatorname{dim}\left(S_{i}\right)$ be the (complex) codimension of $S_{i}$. Let $\left(C_{k}(X)\right)_{k \in \mathbb{Z}}$ be the complex of simplicial chains on $X$ with coefficients in a commutative ring $A$. The complex of intersection chains $\left(I C_{k}(X)\right)_{k \in \mathbb{Z}}$ is the subcomplex of $\left(C_{k}(X)\right)_{k \in Z}$ consisting of chains $c \in C_{k}(X)$ satisfying the allowability condition : For every $i \in I$, the real dimension of $c \cap S_{i}$ is less than $k-c_{i}$, and the real dimension of $\partial c \cap S_{i}$ is less than $k-1-c_{i}$. The intersection homology groups 
$\mathrm{IH}_{k}(X)$ of $X$ are the homology groups of $\left(I C_{k}(X)\right)_{k \in \mathbb{Z}}$. (Note that this is the definition of middle-perversity intersection homology. We can get other interesting intersection homology groups of $X$ by playing with the bounds in the definition of intersection chains, but they will not satisfy Poincaré duality.)

Intersection homology groups satisfy many of the properties of ordinary singular homology groups $\mathrm{H}_{k}(X)$ on smooth varieties. Here are a few of these properties :

- They depend only on $X$, and not on the stratification $\left(S_{i}\right)_{i \in I}$.

- If $X$ is smooth, then $\mathrm{IH}_{k}(X)=\mathrm{H}_{k}(X)$.

- If $X$ is compact, then the $\mathrm{IH}_{k}(X)$ are finitely generated.

- If the coefficients $A$ are a field, the intersection homology groups satisfy the Künneth theorem.

- If $U \subset X$ is open, then there are relative intersection homology groups $\mathrm{IH}_{k}(X, U)$ and an excision long exact sequence.

- It is possible to define an intersection product on intersection homology, and, if $X$ is compact and $A$ is a field, this will induce a nondegenerate linear pairing

$$
\mathrm{IH}_{k}(X) \times \mathrm{IH}_{2 n-k}(X) \longrightarrow A
$$

(I.e., there is a Poincaré duality theorem for intersection homology.)

- Intersection homology satisfies the Lefschetz hyperplane theorem and the hard Lefschetz theorem (if $A$ is a field for hard Lefschetz).

Note however that the intersection homology groups are not homotopy invariants (though they are functorial for certain maps of varieties, called placid maps).

\section{2. $L^{2}$ cohomology of Shimura varieties and intersection ho-} mology. Consider again a Shimura variety $S^{\mathrm{K}}(\mathbb{C})$ as in section 1 (or rather, the complex manifold of its complex points). For every $k \geq 0$, we write $\Omega_{(2)}^{k}\left(S^{\mathrm{K}}(\mathbb{C})\right)$ for the space of smooth forms $\omega$ on $S^{\mathrm{K}}(\mathbb{C})$ such that $\omega$ and $d \omega$ are $L^{2}$. The $L^{2}$ cohomology groups $\mathrm{H}_{(2)}^{*}\left(S^{\mathrm{K}}(\mathbb{C})\right)$ of $S^{\mathrm{K}}(\mathbb{C})$ are the cohomology groups of the complex $\Omega_{(2)}^{*}$. These groups are known to be finite-dimensional and to satisfy Poincaré duality, and in fact we have the following theorem (remember that $\bar{S}^{\mathrm{K}}$ is the Baily-Borel compactification of $S^{\mathrm{K}}$ ) :

Theorem 2.1. There are isomorphisms

$$
\mathrm{H}_{(2)}^{k}\left(S^{\mathrm{K}}(\mathbb{C})\right) \simeq \mathrm{IH}_{2 d-k}\left(\bar{S}^{\mathrm{K}}(\mathbb{C}), \mathbb{R}\right),
$$

where $d=\operatorname{dim}\left(S^{\mathrm{K}}\right)$. Moreover, these isomorphisms are equivariant under the action of $\mathcal{H}_{\mathrm{K}}(\mathbb{R})$. (The Hecke algebra acts on intersection homology because the Hecke correspondences extend to the Baily-Borel compactifications and are still finite, hence placid.) 
This was conjectured by Zucker in [67], and then proved (independently) by Looijenga ([44]), Saper-Stern ([61]) and Looijenga-Rapoport ([45]).

So now we have some things in favour of intersection homology of the BailyBorel compactification : it satisfies Poincaré duality and is isomorphic to a natural invariant of the Shimura variety. We will now see another reason why $L^{2}$ cohomology of Shimura varieties (hence, intersection homology of their Baily-Borel compactification) is easier to study than ordinary cohomology : it is closely related to automorphic representations of the group G. (Ordinary cohomology of Shimura varieties, or cohomology with compact support, is also related to automorphic representations, but in a much more complicated way, see the article [22] of Franke.)

\section{3. $L^{2}$ cohomology of Shimura varieties and discrete auto-} morphic representations. For an introduction to automorphic forms, we refer to the article [10] of Borel and Jacquet and the article [54] of PiatetskiShapiro. Let $\mathbb{A}=\mathbb{A}_{f} \times \mathbb{R}$ be the ring of adeles of $\mathbb{Q}$. Very roughly, an automorphic form on $\mathbf{G}$ is a smooth function $f: \mathbf{G}(\mathbb{A}) \longrightarrow \mathbb{C}$, left invariant under $\mathbf{G}(\mathbb{Q})$, right invariant under some open compact subgroup of $\mathbf{G}\left(\mathbb{A}_{f}\right), \mathrm{K}_{\infty}$-finite on the right (i.e., such that the right translates of $f$ by elements of $\mathrm{K}_{\infty}$ generate a finite dimensional vector space; remember that $\mathrm{K}_{\infty}$ is a maximal compact subgroup of $\mathbf{G}(\mathbb{R})$ ) and satisfying certain growth conditions. The group $\mathbf{G}(\mathbb{A})$ acts on the space of automorphic forms by right translations on the argument. Actually, we are cheating a bit here. The group $\mathbf{G}\left(\mathbb{A}_{f}\right)$ does act that way, but $\mathbf{G}(\mathbb{R})$ does not; the space of automorphic forms is really a Harish-Chandra $\left(\mathfrak{g}, \mathrm{K}_{\infty}\right)$-module, where $\mathfrak{g}$ is the Lie algebra of $\mathbf{G}(\mathbb{C})$. An automorphic representation of $\mathbf{G}(\mathbb{A})$ (or, really, $\left.\mathbf{G}\left(\mathbb{A}_{f}\right) \times\left(\mathfrak{g}, \mathrm{K}_{\infty}\right)\right)$ is an irreducible representation that appears in the space of automorphic forms as an irreducible subquotient.

Note that there is also a classical point of view on automorphic forms, where they are seen as smooth functions on $\mathbf{G}(\mathbb{R})$, left invariant by some arithmetic subgroup of $\mathbf{G}(\mathbb{Q}), \mathrm{K}_{\infty}$-finite on the right and satisfying a growth condition. From that point of view, it may be easier to see that automorphic forms generalize classical modular forms (for modular forms, the group $\mathbf{G}$ is $\mathbf{G} \mathbf{L}_{2}$ ). The two points of view are closely related, cf. [10] 4.3 (in much the same way that the classical and adelic points of view on Shimura varieties are related). In this article, we adopt the adelic point of view, because it makes it easier to see the action of Hecke operators.

Actually, as we are interested only in discrete automorphic representations (see below for a definition), we can see automorphic forms as $L^{2}$ functions on $\mathbf{G}(\mathbb{Q}) \backslash \mathbf{G}(\mathbb{A})$. We follow Arthur's presentation in [1]. First, a word of warning : the quotient $\mathbf{G}(\mathbb{Q}) \backslash \mathbf{G}(\mathbb{A})$ does not have finite volume. This is due to the presence of factors isomorphic to $\mathbb{R}_{>0}$ in the center of $\mathbf{G}(\mathbb{R})$. As in 1.1, let $A_{\infty}=\mathbf{A}(\mathbb{R})^{0}$, where $\mathbf{A}$ is the maximal $\mathbb{R}$-split torus in the center of $\mathbf{G}$. Then $\mathbf{G}(\mathbb{Q}) \backslash \mathbf{G}(\mathbb{A}) / A_{\infty}$ does have finite volume, and we will consider $L^{2}$ functions on this quotient, instead of $\mathbf{G}(\mathbb{Q}) \backslash \mathbf{G}(\mathbb{A})$.

So let $\xi: \mathrm{A}_{\infty} \longrightarrow \mathbb{C}^{\times}$be a character (not necessarily unitary). Then $\xi$ extends 
to a character $\mathbf{G}(\mathbb{A}) \longrightarrow \mathbb{C}^{\times}$, that we will still denote by $\xi$ (cf. I.3 of Arthur's introduction to the trace formula, $[2])$. Let $L^{2}(\mathbf{G}(\mathbb{Q}) \backslash \mathbf{G}(\mathbb{A}), \xi)$ be the space of measurable functions $f: \mathbf{G}(\mathbb{Q}) \backslash \mathbf{G}(\mathbb{A}) \longrightarrow \mathbb{C}$ such that :

(1) for every $z \in \mathrm{A}_{\infty}$ and $g \in \mathbf{G}(\mathbb{A}), f(z g)=\xi(z) f(g)$;

(2) the function $\xi^{-1} f$ is square-integrable on $\mathbf{G}(\mathbb{Q}) \backslash \mathbf{G}(\mathbb{A}) / \mathrm{A}_{\infty}$.

Then the group $\mathbf{G}(\mathbb{A})$ acts on $L^{2}(\mathbf{G}(\mathbb{Q}) \backslash \mathbf{G}(\mathbb{A}), \xi)$ by right translations on the argument. By definition, a discrete automorphic representation of $\mathbf{G}$ is an irreducible representation of $\mathbf{G}(\mathbb{A})$ that appears as a direct summand in $L^{2}(\mathbf{G}(\mathbb{Q}) \backslash$ $\mathbf{G}(\mathbb{A}), \xi)$. It is known that the multiplicity of a discrete automorphic representation $\pi$ in $L^{2}(\mathbf{G}(\mathbb{Q}) \backslash \mathbf{G}(\mathbb{A}), \xi)$ is always finite; we denote it by $m(\pi)$. We also denote by $\Pi_{\text {disc }}(\mathbf{G}, \xi)$ the set of discrete automorphic representations on which $\mathrm{A}_{\infty}$ acts by $\xi$. For the fact that discrete automorphic representations are indeed automorphic representations in the previous sense, see [10] 4.6. (The attentive reader will have noted that automorphic representations are not actual representations of $\mathbf{G}(\mathbb{A})$ because $\mathbf{G}(\mathbb{R})$ does not act on them - while discrete automorphic representations are. How to make sense of our statement that discrete automorphic representations are automorphic is also explained in [10] 4.6.)

Now, given the definition of discrete automorphic representations and the fact that $S^{\mathrm{K}}(\mathbb{C})=\mathbf{G}(\mathbb{Q}) \backslash \mathbf{G}(\mathbb{A}) /\left(\mathrm{A}_{\infty} \mathrm{K}_{\infty} \times \mathrm{K}\right)$, it is not too surprising that the $L^{2}$ cohomology of the Shimura variety $S^{\mathrm{K}}(\mathbb{C})$ should be related to discrete automorphic representations. Here is the precise relation :

Theorem 2.2. (Borel-Casselman, cf. [9] theorem 4.5) Let $\mathrm{K}$ be an open compact subgroup of $\mathbf{G}\left(\mathbb{A}_{f}\right)$. Then there is a $\mathcal{H}_{\mathrm{K}}(\mathbb{C})$-equivariant isomorphism

$$
\mathrm{H}_{(2)}^{*}\left(S^{\mathrm{K}}(\mathbb{C})\right) \otimes_{\mathbb{R}} \mathbb{C} \simeq \bigoplus_{\pi \in \Pi_{d i s c}(\mathbf{G}, 1)} \mathrm{H}^{*}\left(\mathfrak{g}, \mathrm{A}_{\infty} \mathrm{K}_{\infty} ; \pi_{\infty}\right)^{m(\pi)} \otimes \pi_{f}^{\mathrm{K}} .
$$

(This is often called Matsushima's formula when $S^{\mathrm{K}}(\mathbb{C})$ is compact.)

We need to explain the notation. First, the "1" in $\Pi_{\text {disc }}(\mathbf{G}, 1)$ stands for the trivial character of $\mathrm{A}_{\infty}$. (We have chosen to work with the constant sheaf on $S^{\mathrm{K}}$, in order to simplify the notation. In general, for a non-trivial coefficient system on $S^{\mathrm{K}}(\mathbb{C})$, other characters of $\mathrm{A}_{\infty}$ would appear.) Let $\pi \in \Pi_{\text {disc }}(\mathbf{G}, 1)$. Then $\pi$ is an irreducible representation of $\mathbf{G}(\mathbb{A})=\mathbf{G}(\mathbb{R}) \times \mathbf{G}\left(\mathbb{A}_{f}\right)$ so it decomposes as a tensor product $\pi_{\infty} \otimes \pi_{f}$, where $\pi_{\infty}$ (resp. $\pi_{f}$ ) is an irreducible representation of $\mathbf{G}(\mathbb{R})$ (resp. $\mathbf{G}\left(\mathbb{A}_{f}\right)$ ). We denote by $\pi_{f}^{\mathrm{K}}$ the space of $\mathrm{K}$-invariant vectors in the space of $\pi_{f}$; it carries an action of the Hecke algebra $\mathcal{H}_{\mathrm{K}}(\mathbb{C})$. Finally, $\mathrm{H}^{*}\left(\mathfrak{g}, \mathrm{A}_{\infty} \mathrm{K}_{\infty} ; \pi_{\infty}\right)$, the $\left(\mathfrak{g}, \mathrm{A}_{\infty} \mathrm{K}_{\infty}\right)$-cohomology of $\pi_{\infty}$ (where $\mathfrak{g}$ is as before the Lie algebra of $\mathbf{G}(\mathbb{C})$ ), is defined in chapter I of the book [12] by Borel and Wallach.

This gives another reason to study the intersection homology of the Baily-Borel compactifications of Shimura varieties : it will give a lot of information about discrete automorphic representations of $\mathbf{G}$. (Even if only about the ones whose infinite part has nontrivial $\left(\mathfrak{g}, \mathrm{A}_{\infty} \mathrm{K}_{\infty}\right)$-cohomology, and that is a pretty strong condition.) 
Note that there is an issue we have been avoiding until now. Namely, in 1.3, we wanted the cohomology theory on the Shimura variety to also have an action of $\operatorname{Gal}(\bar{E} / E)$, where $E$ is the reflex field (i.e., the field over which the varieties $S^{\mathrm{K}}$ have canonical models). It is not clear how to endow the $L^{2}$ cohomology of $S^{\mathrm{K}}(\mathbb{C})$ with such an action. As we will see in the next section, this will come from the isomorphism of $\mathrm{H}_{(2)}^{*}\left(S^{\mathrm{K}}(\mathbb{C})\right)$ with the intersection homology of $\bar{S}^{\mathrm{K}}(\mathbb{C})$ and from the sheaf-theoretic interpretation of intersection homology (because this interpretation will also make sense in an étale $\ell$-adic setting).

\section{Intersection (co)homology and perverse sheaves}

We use again the notation of section 2 .

3.1. The sheaf-theoretic point of view on intersection homology. Intersection homology of $X$ also has a sheaf-theoretical interpretation. (At this point, we follow Goresky and MacPherson and shift from the homological to the cohomological numbering convention.) For every open $U$ in $X$, let $\mathbf{I} \mathbf{C}^{k}(U)$ be the group of $(2 n-k)$-dimensional intersection chains on $U$ with closed support. If $U^{\prime} \subset U$, then we have a map $\mathbf{I} \mathbf{C}^{k}(U) \longrightarrow \mathbf{I C}^{k}\left(U^{\prime}\right)$ given by restriction of chains. In this way, we get a sheaf $\mathbf{I} \mathbf{C}^{k}$ on $X$. Moreover, the boundary maps of the complex of intersection chains give maps of sheaves $\delta: \mathbf{I C}^{k} \longrightarrow \mathbf{I C}^{k+1}$ such that $\delta \circ \delta=0$, so the $\mathbf{I} \mathbf{C}^{k}$ form a complex of sheaves $\mathbf{I} \mathbf{C}^{*}$ on $X$. This is the intersection complex of $X$. Its cohomology with compact support gives back the intersection homology groups of $X$ :

$$
\mathrm{H}_{c}^{k}\left(X, \mathbf{I C}^{*}(X)\right)=\mathrm{IH}_{2 n-k}(X) .
$$

Its cohomology groups $\operatorname{IH}^{k}(X):=\mathrm{H}^{k}\left(X, \mathbf{I C}^{*}(X)\right)$ are (by definition) the intersection cohomology groups of $X$.

3.2. Perverse sheaves. This point of view has been extended and generalized by the invention of perverse sheaves. The author's favourite reference for perverse sheaves is the book by Beilinson, Bernstein and Deligne ([6]).

To simplify, assume that the ring of coefficients $A$ is a field. Let $D(X)$ be the derived category of the category of sheaves on $X$. This category is obtained from the category of complexes of sheaves on $X$ modulo homotopy by introducing formal inverses of all the quasi-isomorphisms, i.e. of all the morphisms of complexes that induce isomorphisms on the cohomology sheaves. (This is a categorical analogue of a ring localization.) Note that the objects of $D(X)$ are still the complexes of sheaves, we just added more morphisms. The homological functors on the category of complexes of sheaves (such as the various cohomology functors and the Ext and Tor functors) give functors on $D(X)$, and a morphism in $D(X)$ is an isomorphism if and only if it is an isomorphism on the cohomology sheaves.

This category $D(X)$ is still a little big, and we will work with the full subcategory $D_{c}^{b}(X)$ of bounded constructible complexes. If $C^{*}$ is a complex of sheaves, 
we will denote its cohomology sheaves by $\mathrm{H}^{k} C^{*}$. Then $C^{*}$ is called bounded if $\mathrm{H}^{k} C^{*}=0$ for $k<<0$ and $k>>0$. It is called constructible if its cohomology sheaves $\mathrm{H}^{k} C^{*}$ are constructible, that is, if, for every $k \in \mathbb{Z}$, there exists a stratification $\left(S_{i}\right)_{i \in I}$ of $X$ (by smooth subvarieties) such that $\mathrm{H}^{k} C_{\mid S_{i}}^{*}$ is locally constant and finitely generated for every $i$.

For every point $x$ of $X$, we denote by $i_{x}$ the inclusion of $x$ in $X$.

Definition 1. A complex of sheaves $C^{*}$ in $D_{c}^{b}(X)$ is called a perverse sheaf if it satisfies the following support and cosuport conditions :

(1) Support : for every $k \in \mathbb{Z}$,

$$
\operatorname{dim}_{\mathbb{C}}\left\{x \in X \mid \mathrm{H}^{k}\left(i_{x}^{*} C^{*}\right) \neq 0\right\} \leq-k .
$$

(2) Cosupport : for every $k \in \mathbb{Z}$,

$$
\operatorname{dim}_{\mathbb{C}}\left\{x \in X \mid \mathrm{H}^{k}\left(i_{x}^{!} C^{*}\right) \neq 0\right\} \leq k .
$$

We denote by $P(X)$ the category of perverse sheaves on $X$.

Remark 2. Let $x \in X$. There is another way to look at the groups $i_{x}^{*} \mathrm{H}^{k} C^{*}$ and $i_{x}^{!} \mathrm{H}^{k} C^{*}$. Choose an (algebraic or analytic) embedding of a neighbourhood of $x$ into an affine space $\mathbb{C}^{p}$, and let $B_{x}$ denote the intersectioon of this neighbourhood and of a small enough open ball in $\mathbb{C}^{p}$ centered at $x$. Then

$$
\begin{aligned}
\mathrm{H}^{k}\left(i_{x}^{*} C^{*}\right) & =\mathrm{H}^{k}\left(B_{x}, C^{*}\right) \\
\mathrm{H}^{k}\left(i_{x}^{!} C^{*}\right) & =\mathrm{H}_{c}^{k}\left(B_{x}, C^{*}\right) .
\end{aligned}
$$

Remark 3. As before, we are only considering one perversity, the middle (or self-dual) perversity. For other perversities (and much more), see [6].

Note that perverse sheaves are not sheaves but complexes of sheaves. However, the category of perverse sheaves satisfies many properties that we expect from a category of sheaves, and that are not true for $D_{c}^{b}(X)$ (or $D(X)$ ). For example, $P(X)$ is an abelian category, and it is possible to glue morphisms of perverse sheaves (more precisely, categories of perverse sheaves form a stack, say on the open subsets of $X$, cf. [6] 2.1.23).

3.3. Intermediate extensions and the intersection complex. Now we explain the relationship with the intersection complex. First, the intersection complex is a perverse sheaf on $X$ once we put it in the right degree. In fact

Proposition 3.1. The intersection complex $\mathbf{I C}^{*}(X)$ is an object of $D_{c}^{b}(X)$ (i.e., it is a bounded complex with constructible cohomology sheaves), and :

(1) For every $k \neq 0$,

$$
\operatorname{dim}_{\mathbb{C}}\left\{x \in X \mid \mathrm{H}^{k}\left(i_{x}^{*} \mathbf{I C}^{*}(X)\right) \neq 0\right\}<n-k .
$$


(2) For every $k \neq 2 n$,

$$
\operatorname{dim}_{\mathbb{C}}\left\{x \in X \mid \mathrm{H}^{k}\left(i_{x}^{!} \mathbf{I C}^{*}(X)\right) \neq 0\right\}<k-n .
$$

(3) If $U$ is a smooth open dense subset of $X$, then $\mathbf{I C}^{*}(X)_{\mid U}$ is quasi-isomorphic (i.e., isomorphic in $\left.D_{c}^{b}(X)\right)$ to the constant sheaf on $U$.

Moreover, the intersection complex is uniquely characterized by these properties (up to unique isomorphism in $D_{c}^{b}(X)$ ).

In particular, $\mathbf{I C}^{*}(X)[n]$ (that is, the intersection complex put in degree $-n$ ) is a perverse sheaf on $X$.

Even better, it turns out that every perverse sheaf on $X$ is, in some sense, built from intersection complexes on closed subvarieties of $X$. Let us be more precise. Let $j: X \longrightarrow Y$ be a locally closed immersion. Then there is a functor $j_{!_{*}}: P(X) \longrightarrow P(Y)$, called the intermediate extension functor, such that, for every perverse sheaf $K$ on $X$, the perverse sheaf $j_{! *} K$ on $Y$ is uniquely (up to unique quasi-isomorphism) characterized by the following conditions :

(1) For every $k \in \mathbb{Z}$,

$$
\left.\operatorname{dim}_{\mathbb{C}}\left\{x \in Y-X \mid \mathrm{H}^{k}\left(i_{x}^{*} j_{!_{*}} K\right)\right) \neq 0\right\}<-k .
$$

(2) For every $k \in \mathbb{Z}$,

$$
\operatorname{dim}_{\mathbb{C}}\left\{x \in Y-X \mid \mathrm{H}^{k}\left(i_{x}^{!} j_{! *} K\right) \neq 0\right\}<k .
$$

(3) $j^{*} j_{! *} K=K$.

Remark 4. Let us explain briefly the name "intermediate extension". Although it is not clear from the way we defined perverse sheaves, there are "perverse cohomology" functors ${ }^{p} \mathrm{H}^{k}: D_{c}^{b}(X) \longrightarrow P(X)$. In fact, it even turns out that $D_{c}^{b}(X)$ is equivalent to the derived category of the abelian category of perverse sheaves (this is a result of Beilinson, cf. [5]). We can use these cohomology functors to define perverse extension functors ${ }^{p} j_{\text {! }}$ and ${ }^{p} j_{*}$ from $P(X)$ to $P(Y)$. (For example, ${ }^{p} j_{!}={ }^{p} \mathrm{H}^{0} j_{\text {! }}$, where $j_{!}: D_{c}^{b}(X) \longrightarrow D_{c}^{b}(Y)$ is the "extension by zero" functor between the derived categories; likewise for $\left.{ }^{p} j_{*}\right)$. It turns out that, from the perverse point of view, the functor $j_{!}: D_{c}^{b}(Y) \longrightarrow D_{c}^{b}(X)$ is right exact and the functor $j_{*}: D_{c}^{b}(Y) \longrightarrow D_{c}^{b}(X)$ is left exact (that, if $K$ is perverse on $X,{ }^{p} \mathrm{H}^{k} j_{!} K=0$ for $k>0$ and ${ }^{p} \mathrm{H}^{k} j_{*} K=0$ for $\left.k<0\right)$. So the morphism of functors $j_{!} \longrightarrow j_{*}$ induces a morphism of functors ${ }^{p} j_{!} \longrightarrow{ }^{p} j_{*}$. For every perverse sheaf $K$ on $X$, we have :

$$
j_{! *} K=\operatorname{Im}\left({ }^{p} j_{!} K \longrightarrow{ }^{p} j_{*} K\right) .
$$

Now we come back to the description of the category of perverse sheaves on $X$. Let $F$ be a smooth connected locally closed subvariety of $X$, and denote by $i_{F}$ its inclusion in $X$. If $\mathcal{F}$ is a locally constant sheaf on $F$, then it is easy to see that $\mathcal{F}[\operatorname{dim} F]$ is a perverse sheaf on $F$; so $i_{F ! *} \mathcal{F}[\operatorname{dim} F]$ is a perverse sheaf on $X$ (it has support in $\bar{F}$, where $\bar{F}$ is the closure of $F$ in $X$ ). If the locally constant sheaf $\mathcal{F}$ happens to be irreducible, then this perverse sheaf is a simple object in $P(X)$. In fact : 
Theorem 3.2. The abelian category $P(X)$ is artinian and noetherian (i.e., every object has finite length), and its simple objects are all of the form $i_{F ! *} \mathcal{F}[\operatorname{dim} F]$, where $F$ is as above and $\mathcal{F}$ is an irreduible locally constant sheaf on $F$.

Finally, here is the relationship with the intersection complex. Let $i_{F}: F \longrightarrow X$ be as above. Then, if $\mathcal{F}$ is the constant sheaf on $F$, the restriction to $\bar{F}$ of the perverse sheaf $i_{F ! *} \mathcal{F}[\operatorname{dim} F]$ is isomorphic to $\operatorname{IC}^{*}(\bar{F})[\operatorname{dim} F]$. In fact, we could define the intersection complex on a (possibly singular) variety $Y$ with coefficients in some locally constant sheaf on the smooth locus of $Y$, and then the simple objects in $P(X)$ would all be intersection complexes on closed subvarieties of $X$.

3.4. $\ell$-adic perverse sheaves. Now we come at last to the point of this section (to make the Galois groups $\mathrm{Gal}(\bar{E} / E)$ act on the intersection (co)homology of $\bar{S}^{\mathrm{K}}(\mathbb{C})$ ).

Note that the definitions of the category of perverse sheaves and of the intermediate extension in 3.2 and 3.3 would work just as well in a category of étale $\ell$-adic sheaves. So now we take for $X$ a quasi-separated scheme of finite type over a field $k$, we fix a prime number $\ell$ invertible in $k$ and we consider the category $D_{c}^{b}\left(X, \overline{\mathbb{Q}}_{\ell}\right)$ of bounded $\ell$-adic complexes on $X$. (To avoid a headache, we will take $k$ to be algebraically closed or finite, so the simple construction of [6] 2.2.14 applies.) Then we can define an abelian subcategory of perverse sheaves $P(X)$ in $D_{c}^{b}\left(X, \overline{\mathbb{Q}}_{\ell}\right)$ and intermediate extension functors $j_{! *}: P(X) \longrightarrow P(Y)$ as before (see [6] 2.2). In particular, we can make the following definition :

Definition 2. Suppose that $X$ is purely of dimension $n$, and let $j: U \longrightarrow X$ be the inclusion of the smooth locus of $X$ in $X$. Then the ( $\ell$-adic) intersection complex of $X$ is

$$
\mathbf{I C}^{*}(X)=\left(j ! * \overline{\mathbb{Q}}_{\ell, U}[n]\right)[-n],
$$

where $\overline{\mathbb{Q}}_{\ell, U}$ is the constant sheaf $\overline{\mathbb{Q}}_{\ell}$ on $U$. The $\ell$-adic intersection cohomology $\mathrm{IH}^{*}\left(X, \overline{\mathbb{Q}}_{\ell}\right)$ of $X$ is the cohomology of $\mathbf{I C}^{*}(X)$.

3.5. Application to Shimura varieties. We know that the Shimura variety $S^{\mathrm{K}}$ and its Baily-Borel compactification $\bar{S}^{\mathrm{K}}$ are defined over the number field $E$. So we can form the $\ell$-adic intersection cohomology groups $\operatorname{IH}^{*}\left(\bar{S}_{\bar{E}}^{\mathrm{K}}, \overline{\mathbb{Q}}_{\ell}\right)$. They admit an action of $\operatorname{Gal}(\bar{E} / E)$. Moreover, if we choose a field isomorphism $\overline{\mathbb{Q}}_{\ell} \simeq \mathbb{C}$, then the comparison theorems between the étale topology and the classical topology will give an isomorphism $\operatorname{IH}^{*}\left(\bar{S}_{\bar{E}}^{\mathrm{K}}, \overline{\mathbb{Q}}_{\ell}\right) \simeq \operatorname{IH}^{*}\left(\bar{S}^{\mathrm{K}}(\mathbb{C}), \mathbb{C}\right)$ (cf. chapter 6 of $[6])$.

The isomorphism of 2.2 between intersection homology of $\bar{S}^{\mathrm{K}}(\mathbb{C})$ and $L^{2}$ cohomology of $S^{\mathrm{K}}(\mathbb{C})$, as well as the duality between intersection homology and intersection cohomology (cf. 3.1), thus give an isomorphism

$$
\mathrm{IH}^{*}\left(\bar{S}_{\bar{E}}^{\mathrm{K}}, \overline{\mathbb{Q}}_{\ell}\right) \simeq \mathrm{H}_{(2)}^{*}\left(S^{\mathrm{K}}(\mathbb{C})\right) \otimes \mathbb{C},
$$


and this isomorphism is equivariant under the action of $\mathcal{H}_{\mathrm{K}}(\mathbb{C})$. We know what $L^{2}$ cohomology looks like as a representation of $\mathcal{H}_{\mathrm{K}}(\mathbb{C})$, thanks to the theorem of Borel and Casselman (cf. 2.3).

Using this theorem and his own trace invariant formula, Arthur has given a formula for the trace of a Hecke operator on $\mathrm{H}_{(2)}^{*}\left(S^{\mathrm{K}}(\mathbb{C})\right) \otimes \mathbb{C}(\mathrm{cf}$. [1]). This formula involves global volume terms, discrete series characters on $\mathbf{G}(\mathbb{R})$ and orbital integrals on $\mathbf{G}\left(\mathbb{A}_{f}\right)$.

The problem now is to understand the action of the Galois group Gal $(\bar{E} / E)$. We have a very precise conjectural description of the intersection cohomology of $\bar{S}^{\mathrm{K}}$ as a $\mathcal{H}_{\mathrm{K}}(\mathbb{C}) \times \operatorname{Gal}(\bar{E} / E)$-module, see for example the articles [34] of Kottwitz and [7] of Blasius and Rogawski.

In the next sections, we will explain a strategy to understand how at least part of the Galois group $\operatorname{Gal}(\bar{E} / E)$ acts.

\section{Counting points on Shimura varieties}

We want to understand the action of the Galois group $\operatorname{Gal}(\bar{E} / E)$ on the intersection cohomology groups $\mathrm{IH}_{\mathrm{K}}^{*}:=\mathrm{IH}^{*}\left(\bar{S}_{\bar{E}}^{\mathrm{K}}, \overline{\mathbb{Q}}_{\ell}\right)$. It is conjectured that this action is unramified almost everywhere. Thus, by the Chebotarev density theorem, it is theoretically enough to understand the action of the Frobenius automorphisms at the places of $E$ where the action is unramified, and one way to do this is to calculate the trace of the powers of the Frobenius automorphisms at these places. However, for some purposes, it is necessary to look at the action of the decomposition groups at other places. This is part of the theory of bad reduction of Shimura varieties, and we will not talk about this here, nor will we attempt to give comprehensive references to it. (Let us just point to the book [31] of Harris and Taylor.)

In general, intersection cohomology can be very hard to calculate. First we will look at simpler objects, the cohomology groups with compact support $\mathrm{H}_{c, \mathrm{~K}}^{*}:=$ $\mathrm{H}_{c}^{*}\left(S_{\bar{E}}^{\mathrm{K}}, \overline{\mathbb{Q}}_{\ell}\right)$. Assume that the Shimura varieties and their compactifications (the Baily-Borel compactifications and the toroidal compactifications) have "good" models over an open subset $U$ of Spec $\mathcal{O}_{E}$, and write $\mathcal{S}^{\mathrm{K}}$ for the model of $S^{\mathrm{K}}$. (It is much easier to imagine what a "good" model should be than to write down a precise definition. An attempt has been made in [49] 1.3, but it is by no means optimal.) Then, by the specialization theorem (SGA 4 III Exposé XVI 2.1), and also by Poincaré duality (cf. SGA 4 III Exposé XVIII), for every finite place $\mathfrak{p}$ of $E$ such that $\mathfrak{p} \in U$ and $\mathfrak{p} \chi \ell$, there is a $\operatorname{Gal}\left(\bar{E}_{\mathfrak{p}} / E_{\mathfrak{p}}\right)$-equivariant isomorphism

$$
\mathrm{H}_{c, \mathrm{~K}}^{*}=\mathrm{H}_{c}^{*}\left(S_{\bar{E}}^{\mathrm{K}}, \overline{\mathbb{Q}}_{\ell}\right) \simeq \mathrm{H}_{c}^{*}\left(\mathcal{S}_{\overline{\mathbb{F}}_{\mathfrak{p}}}^{\mathrm{K}}, \overline{\mathbb{Q}}_{\ell}\right),
$$

where $\mathbb{F}_{\mathfrak{p}}$ is the residue field of $\mathcal{O}_{E}$ at $\mathfrak{p}$. In particular, the $\operatorname{Gal}(\bar{E} / E)$-representation $\mathrm{H}_{c, \mathrm{~K}}^{*}$ is unramified at $\mathfrak{p}$.

Now, by Grothendieck's fixed point formula (SGA 4 1/2 Rapport), calculating the trace of powers of the Frobenius automorphism on $\mathrm{H}_{c}^{*}\left(\mathcal{S}_{\overline{\mathbb{F}}_{\mathrm{p}}}^{\mathrm{K}}, \overline{\mathbb{Q}}_{\ell}\right)$ is the same as counting the points of $\mathcal{S}^{\mathrm{K}}$ over finite extensions of $\mathbb{F}_{\mathfrak{p}}$. 
Langlands has given a conjectural formula for this number of points, cf. [40] and [34]. Ihara had earlier made and proved a similar conjecture for Shimura varieties of dimension 1. Although this conjecture is not known in general, it is easier to study for a special class of Shimura varieties, the so-called PEL Shimura varieties. These are Shimura varieties that can be seen as moduli spaces of abelian with certain supplementary structures ( $\mathrm{P}$ : polarizations, $\mathrm{E}$ : endomorphisms, i.e. complex multiplication by certain $\mathrm{CM}$ number fields, and $\mathrm{L}:$ level structures). For PEL Shimura varieties of types $A$ and $C$ (i.e., such that the group $\mathbf{G}$ is of type $A$ or $C$ ), Langlands's conjecture had been proved by Kottwitz in [35]. Note that all the examples we gave in 1.1 are of this type. Conveniently enough, the modular interpretation of PEL Shimura varieties also gives a model of the Shimura variety over an explicit open subset of $\operatorname{Spec} \mathcal{O}_{E}$.

In fact, Kottwitz has done more than counting points; he has also counted the points that are fixed by the composition of a power of the Frobenius automorphism and of a Hecke correspondence (with a condition of triviality at $\mathfrak{p}$ ). So, using Deligne's conjecture instead of Grothendieck's fixed point formula, we can use Kottwitz's result to understand the commutating actions of $\mathrm{Gal}(\bar{E} / E)$ and of $\mathcal{H}_{\mathrm{K}}\left(\overline{\mathbb{Q}}_{\ell}\right)$ on $\mathrm{H}_{c, \mathrm{~K}}^{*}$. (Deligne's conjecture gives a simple formula for the local terms in the Lefschetz fixed formula if we twist the correspondence by a high power of the Frobenius. It is now a theorem and has been proved independently by Fujiwara in [23] and Varshavsky in [63]. In the case of Shimura varieties, it also follows from an earlier result of Pink in [57].)

Using his counting result, Kottwitz has proved the conjectural description of $\mathrm{IH}_{\mathrm{K}}^{*}$ for some simple Shimura varieties (cf. [36]). Here "simple" means that the Shimura varieties are compact (so intersection cohomology is cohomology with compact support) and that the phenomenon called "endoscopy" (about which we are trying to say as little as possible) does not appear.

One reason to avoid endoscopic complications was that a very important and necessary result when dealing with endoscopy, the so-called "fundamental lemma", was not available at the time. It now is, thanks to the combined efforts of many people, among which Kottwitz ([33]), Clozel ([15]), Labesse ([38], [16]), Hales ([30]), Laumon, Ngo ([43], [53]), and Waldspurger ([64], [65], [66]).

Assuming the fundamental lemma, the more general case of compact PEL Shimura varieties of type $A$ or $C$ (with endoscopy playing a role) was treated by Kottwitz in [34], admitting Arthur's conjectures on the descripton of discrete automorphic representations of G. Actually, Kottwitz did more : he treated the case of the (expected) contribution of $\mathrm{H}_{c, \mathrm{~K}}^{*}$ to $\mathrm{IH}_{\mathrm{K}}^{*}$. Let us say a word about Arthur's conjectures. Arthur has announced a proof of a suitable formulation of his conjectures for classical groups (that is, symplectic and orthogonal groups), using the stable twisted trace formula. His proof is expected to adapt to the case of unitary groups (that is, the groups that give PEL Shimura varieties of type $A$ ), but this adaptation will likely require a lot of effort.

Let us also note that the case of compact PEL Shimura varieties of type $A$ should be explained in great detail in the book project led by Michael Harris ([8]).

This does not tell us what to do in the case where $S^{\mathrm{K}}$ is not projective. First 
note that the modular interpretation gives us integral models of the Shimura varieties but not of their compactifications. So this is the first problem to solve. Fortunately, it has been solved : See the article [21] of Deligne and Rapoport for the case of modular curves, the book [14] by Chai and Faltings for the case of Siegel modular varieties, Larsen's article [42] for the case of Picard modular varieties, and Lan's dissertation [39] for the general case of PEL Shimura varieties of type $A$ or $C$. This allows us to apply the specialization theorem to intersection cohomology. In particular, we get the fact that the $\mathrm{Gal}(\bar{E} / E)$-representation $\mathrm{IH}_{c, \mathrm{~K}}^{*}$ is unramified almost everywhere, and, at the finite places $\mathfrak{p}$ where it is unramified, we can study it by considering the reduction modulo $\mathfrak{p}$ of the Shimura variety and its compactifications.

Next we have to somehow describe the intersection complex. If the group $\mathbf{G}$ has semi-simple $\mathbb{Q}$-rank 1 , so it has only one conjugacy class of rational parabolic subgroups, then the Baily-Borel compactification is simpler (it only has one kind of boundary strata) and we can obtain the intersection complex by a simple truncation process from the direct image on $\bar{S}^{\mathrm{K}}$ of the constant sheaf on $S^{\mathrm{K}}$. The conjectural description of $\mathrm{IH}_{\mathrm{K}}^{*}$ is know for the cases $\mathbf{G}=\mathbf{G L}_{2}$ (see the book [20]) and the case of Picard modular surfaces, i.e., $\mathbf{G}=\mathbf{G} \mathbf{U}(2,1)$ (see the book [41]). In the general case of semi-simple $\mathbb{Q}$-rank 1 , Rapoport has given in [58] a formula for the trace of a power of the Frobenius automorphism (at almost every place) on the stalks of the intersection complex.

In the general case, the intersection complex is obtained from the direct image of the constant sheaf on $S^{\mathrm{K}}$ by applying several nested truncations (cf. [6] 2.1.11), and it is not clear how to see the action of Frobenius on the stalks of this thing. We will describe a solution in the next section.

\section{Weighted cohomology}

In this section, $j$ will be the inclusion of $S^{\mathrm{K}}$ in its Baily-Borel compactification $\bar{S}^{\mathrm{K}}$, and $j_{*}$ will be the derived direct image functor. Here is the main idea : instead of seeing the intersection complex $I C^{*}\left(\bar{S}^{\mathrm{K}}\right)$ as a truncation of $j_{*} \overline{\mathbb{Q}}_{\ell, S^{\mathrm{K}}}$ by the cohomology degree (on various strata of $\bar{S}^{\mathrm{K}}-S^{\mathrm{K}}$ ), we want to see it as a truncation by Frobenius weights (in the sense of Deligne). This idea goes back to the construction by Goresky, Harder and MacPherson of the weighted cohomology complexes in a topological setting (i.e., on a non-algebraic compactification of the set of complex points $\left.S^{\mathrm{K}}(\mathbb{C})\right)$.

5.1. The topological case. As we have mentioned before, the manifold $S^{\mathrm{K}}(\mathbb{C})$ has a lot of non-algebraic compactifications (these compactifications are defined for a general locally symmetric space, and not just for a Shimura variety). The one used in the construction of weighted cohomology is the reductive Borel-Serre compactification $S^{\mathrm{K}}(\mathbb{C})^{R B S}$ (cf. [11] III.6 and III.10; the reductive Borel-Serre compactification was originally defined by Zucker in [67], though 
not under that name). The reductive Borel-Serre compactification admits a map $\pi: S^{\mathrm{K}}(\mathbb{C})^{R B S} \longrightarrow \bar{S}^{\mathrm{K}}(\mathbb{C})$ that extends the identity on $S^{\mathrm{K}}(\mathbb{C})$; we also denote by $\widetilde{j}$ the inclusion of $S^{\mathrm{K}}(\mathbb{C})$ in $S^{\mathrm{K}}(\mathbb{C})^{R B S}$.

The boundary $S^{\mathrm{K}}(\mathbb{C})^{R B S}-S^{\mathrm{K}}(\mathbb{C})$ of $S^{\mathrm{K}}(\mathbb{C})^{R B S}$ has a very pleasant description. It is a union of strata, each of which is a locally symmetric space for the Levi quotient of a rational parabolic subgroup of $\mathbf{G}$; moreover, the closure of a stratum is its reductive Borel-Serre compactification. (A lot more is known about the precise geometry of the strata, see, e.g., [27] 1D).

The weighted cohomology complexes are bounded constructible complexes $W^{\mu}$ of $\mathbb{C}$ or $\mathbb{Q}$-vector spaces on $S^{\mathrm{K}}(\mathbb{C})^{R B S}$ extending the constant sheaf on $S^{\mathrm{K}}(\mathbb{C})$, constructed by Goresky, Harder and MacPherson in [27] (they give two constructions, one for $\mathbb{C}$-coefficients and one for $\mathbb{Q}$-coefficients, and then show that the two constructions agree). They depend on a weight profile $\mu$ (which is a function from the set of relative simple roots of $\mathbf{G}$ to $\mathbb{Z}+\frac{1}{2}$ ). The basic idea of weighted cohomology is to consider the complex $\widetilde{j}_{*} \mathbb{C}\left(\right.$ or $\widetilde{j}_{*} \mathbb{Q}$ ) on $S^{\mathrm{K}}(\mathbb{C})^{R B S}$ and to truncate it, not by the cohomology degree as for the intersection complex, but by the weights of certain tori. More precisely, on a strata $S$ corresponding to a Levi subgroup $\mathbf{M}$, we truncate by the weights of the $\mathbb{Q}$-split torus $\mathbf{A}_{M}$ in the center of $\mathbf{M}$ (the group $\mathbf{A}_{M}(\mathbb{Q})$ acts on $\widetilde{j}_{*} \mathbb{C}_{\mid S}$ by what Goresky, Harder and MacPherson call Looijenga Hecke correspondences). The weight profile specifies, for every strata, which weights to keep.

Of course, it is not that simple. The complex $\widetilde{j}_{*} \mathbb{C}$ is an object in a derived category (which is not abelian but triangulated), and it is not so easy to truncate objects in such a category. To get around this problem, the authors of [27] construct an incarnation of $\widetilde{j}_{*} \mathbb{C}$, that is, an explicit complex that is quasi-isomorphic to $\widetilde{j} * \mathbb{C}$ and on which the tori $\mathbf{A}_{M}(\mathbb{Q})$ still act. (In fact, they construct two incarnations, one of $\widetilde{j}_{*} \mathbb{C}$ and one of $\widetilde{j}_{*} \mathbb{Q}$ ).

The upshot (for us) is that the functor $\pi_{*}: D_{c}^{b}\left(S^{\mathrm{K}}(\mathbb{C})^{R B S}\right) \longrightarrow D_{c}^{b}\left(\bar{S}^{\mathrm{K}}(\mathbb{C})\right)$ sends two of these weighted cohomology complexes to the intersection complex on $\bar{S}^{\mathrm{K}}(\mathbb{C})$ (they are the complexes corresponding to the lower and upper middle weight profiles). On the other hand, the weighted cohomology complexes are canonical enough so that the Hecke algebra acts on their cohomology, and explicit enough so that it is possible to calculate the local terms when we apply the Lefschetz fixed point formula to them. This is possible but by no means easy, and is the object of the article [26] of Goresky and MacPherson. Then, in the paper [29], Goresky, Kottwitz and MacPherson show that the result of [26] agrees with the result of Arthur's calculation in [1].

The problem, from our point of view, is that this construction is absolutely not algebraic, so it is unclear how to use it to understand the action of $\operatorname{Gal}(\bar{E} / E)$ on $\mathrm{IH}^{*}\left(S^{\mathrm{K}}, \overline{\mathbb{Q}}_{\ell}\right)$.

Remark 5. There is another version of weighted cohomology of locally symmetric spaces : Franke's weighted $L^{2}$ cohomology, defined in [22]. In his article [52], Nair has shown that Franke's weighted $L^{2}$ cohomology groups are weighted cohomology groups in the sense of Goresky-Harder-MacPherson. 
5.2. Algebraic construction of weighted cohomology. First, the reductive Borel-Serre compactification is not an algebraic variety, so what we are really looking for is a construction of the complexes $\pi_{*} W^{\mu}$, directly on the BailyBorel compactification. This looks difficult for several reasons. The Baily-Borel compactification is very singular, which is one of the reasons why Goresky, Harder and MacPherson use the less singular reductive Borel-Serre compactification in the first place. Besides, the boundary strata in $\bar{S}^{\mathrm{K}}$ correspond to maximal rational parabolic subgroups of $\mathbf{G}$, and several strata in $S^{\mathrm{K}}(\mathbb{C})^{R B S}$ can be (rather brutally) contracted to the same stratum in $\bar{S}^{\mathrm{K}}(\mathbb{C})$. It is possible to give a description of the stalks of $\pi_{*} W^{\mu}$ (see the article [28] of Goresky, Harder, MacPherson and Nair), but it is a rather complicated description, much more complicated than the simple description of the stalks of $W^{\mu}$.

The idea is that the action of the Looijenga Hecke correspondences should correspond in some way to the action of the Frobenius automorphism in an algebraic setting. This is actually a very natural ideal. Looijenga himself uses the fact that the eigenspaces of the Looijenga Hecke correspondences are pure in the sense of mixed Hodge theory (cf. [44] 4.2), and we know that the weight filtration of Hodge theory corresponds to the filtration by Frobenius weights in $\ell$-adic cohomology (cf. for example [6] 6.2.2). So the correct algebraic analogue of the truncations of [27] should be a truncation by Frobenius weights (in the sense of Deligne's [19], see also chapter 5 of [6]). As a consequence, the most natural place to define the algebraic analogues of the weighted cohomology complexes is the reduction modulo $\mathfrak{p}$ of an integral model of $\bar{S}^{\mathrm{K}}$, where $\mathfrak{p}$ is a finite place of $E$ where good integral models exist. (But see the remark at the end of this subsection.)

In fact, it turns out that we can work in a very general setting. Let $\mathbb{F}_{q}$ be a finite field, and $X$ be a quasi-separated scheme of finite type over $\mathbb{F}_{q}$. Then we have the category of mixed $\ell$-adic complexes $D_{m}^{b}\left(X, \overline{\mathbb{Q}}_{\ell}\right)$ on $X$, cf. [6] 5.1. (Here "mixed" refers to the weights of the complexes, and the weights are defined by considering the action of the Frobenius automorphisms on the stalks of the complexes; for more details, see [19] or [6] 5). In particular, we get a category $P_{m}(X)$ of mixed $\ell$-adic perverse sheaves on $X$ as a subcategory of $D_{m}^{b}\left(X, \overline{\mathbb{Q}}_{\ell}\right)$. One important result of the theory is that mixed perverse sheaves admit a canonical weight filtration. That is, if $K$ is an object in $P_{m}(X)$, then it has a canonical filtration $\left(w_{\leq a} K\right)_{a \in \mathbb{Z}}$ such that each $w_{\leq a} K$ is a subperverse sheaf of $K$ of weight $\leq a$ and such that $K / w_{\leq a} K$ is of weight $>a$.

This functor $w_{\leq a}$ on mixed perverse sheaves does not extend to $D_{m}^{b}\left(X, \overline{\mathbb{Q}}_{\ell}\right)$ in the naïve way; that is, the inclusion functor from the category of mixed sheaves of weight $\leq a$ to $D_{m}^{b}\left(X, \overline{\mathbb{Q}}_{\ell}\right)$ does not admit a right adjoint. But we can extend $w_{\leq a}$ in another way. Consider the full subcategory ${ }^{w} D^{\leq a}$ of $D_{m}^{b}\left(X, \overline{\mathbb{Q}}_{\ell}\right)$ whose objects are the complexes $K$ such that, for every $k \in \mathbb{Z}$, the $k$-th perverse cohomology sheaf ${ }^{p} \mathrm{H}^{k} K$ is of weight $\leq a$. (If we wanted to define the complexes of weight $\leq a$, we would require ${ }^{p} \mathrm{H}^{k} K$ to be of weight $\leq a+k$.) Then ${ }^{w} D^{\leq a}$ is a triangulated subcategory of $D_{m}^{b}\left(X, \overline{\mathbb{Q}}_{\ell}\right)$, and the inclusion ${ }^{w} D^{\leq a} \subset D_{m}^{b}\left(X, \overline{\mathbb{Q}}_{\ell}\right)$ does admit a right adjoint, which we denote by $w_{\leq a}$ (because it extends the previous $w_{\leq a}$ ). Likewise, we can define a full triangulated subcategory ${ }^{w} D^{\geq a}$ of $D_{m}^{b}\left(X, \overline{\mathbb{Q}}_{\ell}\right)$, whose 
inclusion into $D_{m}^{b}\left(X, \overline{\mathbb{Q}}_{\ell}\right)$ admits a left adjoint $w_{\geq a}$ (extending the functor $K \longmapsto$ $K / w_{\leq a-1} K$ on mixed perverse sheaves). This is explained in section 3 of [48]. Then the analogue of the theorem that $\pi_{*} W^{\mu}$ is the intersection complex (for a well-chosen weight profile $\mu$ ) is the :

Theorem 5.1. ([48] 3.1.4) Let $j: U \longrightarrow X$ a nonempty open subset of $X$ and $K$ be a pure perverse sheaf of weight a on $U$. Then there are canonical isomorphisms :

$$
j_{! *} K \simeq w_{\leq a} j_{*} K \simeq w_{\geq a} j_{!} K .
$$

More generally, if we have a stratification on $X$, we can choose to truncate by different weights on the different strata (cf. [48] 3.3); in this way, we get analogues of the other weighted cohomology complexes, or rather of their images on the Baily-Borel compactification. We also get somewhat more explicit formulas for $w_{\leq a}$, and hence the intersection complex ([48] 3.3.4 and 3.3.5), analogous to the formula of [6] 2.1.11, but where all the truncations by the cohomology degree have been replaced by weight truncations. The reason this makes such a big difference is that the weight truncation functors $w_{\leq a}$ and $w_{\geq a}$ are exact in the perverse sense. (Interestingly enough, it turns out that, in this setting, the weighted cohomology complexes are canonically defined and have nothing to do with Shimura varieties. In fact, there is another application of these ideas, to Schubert varieties, see [50].)

Remark 6. We want to make a remark about the construction of the weighted cohomology complexes on the canonical models $\bar{S}^{\mathrm{K}}$ (and not their reduction modulo a prime ideal). The construction of [48] 3 is very formal and will apply in every category that has a notion of weights and a weight truncation on "perverse" objects. For example, it should apply without any changes to Saito's derived category of mixed Hodge modules. In fact, Arvind Nair has just informed the author that he has indeed been able to construct weighted cohomology complexes in the category of mixed Hodge modules, and to prove that the weighted cohomology complexes he obtained on the Baily-Borel compactification of a Shimura variety are the pushforwards of the Goresky-Harder-MacPherson weighted cohomology complexes on the reductive Borel-Serre compactification. As an application of this, he was able to prove that Franke's spectral sequence ([22] 7.4) is a spectral sequence of mixed Hodge structures (for the locally symmetric spaces that are Shimura varieties).

Now suppose that $X$ is a quasi-separated scheme of finite type over a number field. We can define $\ell$-adic perverse sheaves on $X$, and we can also define a notion of weights for $\ell$-adic complexes on $X$ (cf. Deligne's [19] 1.2.2 and Huber's article [32]). The problem is that mixed perverse sheaves on $X$ do not have a weight filtration in general (because number fields have more Galois cohomology than finite fields). To circumvent this problem, we could try to work in the derived category of the abelian category of mixed perverse sheaves on $X$ admitting a weight filtration. Then it is not obvious how to construct the $4 / 5 / 6$ operations on these categories. It might be possible to copy Saito's approach in [59] (where he constructs and studies the derived category of mixed Hodge modules); see also Saito's preprint [60]. As far as the author knows, this has not been worked out anywhere. 
5.3. Application to the cohomology of Shimura varieties. Once we have the interpretation of the intermediate extension functor given in the previous subsection, it becomes surprisingly easy to calculate the trace of Frobenius automorphisms on the stalks of $\mathbf{I C}^{*}\left(\bar{S}^{\mathrm{K}}\right)$. We should mention that one reason it is so easy is that one of the main ingredients, a description of the restriction to the boundary strata of the complex $j_{*} \overline{\mathbb{Q}}_{\ell}$ (where $j$ is again the inclusion of $S^{\mathrm{K}}$ in $\bar{S}^{\mathrm{K}}$ ) has been provided by Pink in [56]. And of course, the whole calculation rests on Kottwitz's calculations for the cohomology with compact support (in [35]). Including Hecke correspondences in the picture is just a matter of bookkeeping, and the final result of the Lefschetz trace formula appears in [49] 1.7.

This is not the end of the story. It still remains to compare the result of the Lefschetz fixed point formula with Arthur's invariant trace formula, in order to try to prove the result conjectured in 10.1 of Kottwitz's article [34]. This is basically a generalization of part I of [34] to include the non-elliptic terms. Given the work done by Kottwitz in [34] and [37], it requires no new ideas, but still takes some effort. In the case of general unitary groups over $\mathbb{Q}$, it is the main object of the book [49] (along with some applications).

Even then, we are not quite done. If we want to prove the conjectural description of $\operatorname{IH}^{*}\left(\bar{S}^{\mathrm{K}}, \overline{\mathbb{Q}}_{\ell}\right)$ given in [34] or [7], we still need to know Arthur's conjectures.

Some applications that do not depend on Arthur's conjectures are worked out in the book [49] (subsection 8.4). They use a weak form of base change from unitary groups to general linear groups, for the automorphic representations that appear in the $L^{2}$ cohomology of Shimura varieties. (If we knew full base change, then we would probably also know Arthur's conjectures.) Let us mention the two main applications :

- The logarithm of the $L$-function of the intersection complex is a linear combination of logarithms of $L$-functions of automorphic representations of general linear groups ([49] corollary 8.4.5). In fact, we can even get similar formulas for the $L$-functions of the $\mathcal{H}_{\mathrm{K}}\left(\overline{\mathbb{Q}}_{\ell}\right)$-isotypical components of the intersection cohomology, as in [49] 7.2.2. However, the coefficients in these linear combinations are not explicit, and in particular [49] does not show that they are integers.

- We can derive some cases of the global Langlands correspondence (cf. [49] $8.4 .9,8.4 .10)$. Note however that one of the conclusions of [49] is that, in the end, we do not get more Galois representations in the cohomology of noncompact unitary varieties than we would in the cohomology of compact unitary Shimura varieties. In particular, the cases of the Langlands correspondence that are worked out in [49] can also be obtained using compact Shimura varieties and gluing of Galois representations (cf. the last chapters of the book project [8] or the article [62] of Shin; note that Shin also considers places of bad reduction). 


\section{References}

[1] J. Arthur, The $L^{2}$-Lefschetz numbers of Hecke operators, Inv. Math. 97 (1989), pp. $257-290$.

[2] J. Arthur, An introduction to the trace formula, in Harmonic analysis, the trace formula, and Shimura varieties, pp. 1-263, Clay Math. Proc., 4, Amer. Math. Soc., Providence, RI, 2005.

[3] A. Ash, D. Mumford, M. Rapoport et Y. Tai, Smooth compactification of locally symmetric spaces, Lie groups : history, frontiers and applications vol. 4 (1975).

[4] W. Baily et A. Borel, Compactification of arithmetic quotients of bounded symmetric domains, Ann. of Math. (2) 84 (1966), pp. 442-528.

[5] A. Beilinson, On the derived category of perverse sheaves, in $K$-theory, arithmetic and geometry (Moscow, 1984-1986), pp. 27-41, Lecture Notes in Math., 1289 (1987).

[6] A. Beilinson, J. Bernstein et P. Deligne, Analyse et topologie sur les espaces singuliers (I), Astérique 100 (1982).

[7] D. Blasius and J. Rogawski, Zeta functions of Shimura varieties, in Motives (Seattle, WA, 1991), pp. 525-571, Proc. Sympos. Pure Math., 55, Part 2, Amer. Math. Soc., Providence, RI, 1994.

[8] Book Project : Stabilisation de la formule des traces, variétés de Shimura, et applications arithmétiques, http://www.institut.math.jussieu.fr/projets/fa/bp0.html

[9] A. Borel and W. Casselman, $L^{2}$-cohomology of locally symmetric manifolds of finite volume, Duke Math. J. 50 (1983), pp. 625-647.

[10] A. Borel and H. Jacquet, Automorphic forms and automorphic representations, in Automorphic forms, representations, and L-functions (Proc. Symposia in Pure Math., volume 33, 1977), part 1, pp. 189-202.

[11] A. Borel and L. Ji, Compactifications of symmetric and locally symmatric spaces, Mathematics: Theory and Applications, Birkäuser (2006).

[12] A. Borel and N. Wallach, Continuous cohomology, discrete subgroups, and representations of reductive groups. Second edition, Mathematical surveys and monographs 57, AMS (2000).

[13] M. Borovoi, Langlands's conjecture concerning conjugation of connected Shimura varieties, Selecta Math. Soviet. 3 (1983/84), no. 1, pp. 3-39.

[14] C.-L. Chai et G. Faltings, Degenerations of abelian varieties, Ergebnisse der Mathematik und ihrer Grenzgebiete 22, Springer (1980).

[15] L. Clozel, The fundamental lemma for stable base change, Duke Math. J. 61 (1990), n1, pp. 255-302.

[16] L. Clozel and J.-P. Labesse, Changement de base pour les représentations cohomologiques de certains groupes unitaires, Astérisque 257 (1999), pp. 119-133.

[17] P. Deligne, Travaux de Shimura, Séminaire Bourbaki, exposé 389, février 1971

[18] P. Deligne, Variétés de Shimura: Interprétation modulaire, et techniques de construction de modèles canoniques, Proc. Sympos. Pure Math., Part 2, 33, pp. 247-290, Amer. Math. Soc., Providence, RI, 1979.

[19] P. Deligne, La conjecture de Weil. II., Publications Mathématiques de l'IHES 52 (1981), pp. 137-251. 
[20] P. Deligne and W. Kuyk (editors), Modular functions of one variable II, Lecture Notes in Mathematics, vol. 349, Springer (1973).

[21] P. Deligne and M. Rapoport, Les schémas de modules de courbes elliptiques, in [20], pp. 143-316.

[22] J. Franke, Harmonic analysis in weighted $L_{2}$-spaces, Ann. Sci. École Norm. Sup. (4) 31 (1998), no. 2, pp. 181-279.

[23] K. Fujiwara, Rigid geometry, Lefschetz-Verdier trace formula and Deligne's conjecture, Invent. Math. 127, pp. 489-533 (1997).

[24] M. Goresky and R. MacPherson, Intersection homology theory, Topology 19 (1980), no. 2, pp. 135-162.

[25] M. Goresky and R. MacPherson, Intersection homology II, Invent. Math. 72 (1983), no. 1 , pp. $77-129$.

[26] M. Goresky and R. MacPherson, The topological trace formula, J. Reine Angew. Math. 560 (2003), pp. 77-150.

[27] M. Goresky, G. Harder and R. MacPherson, Weighted cohomology, Invent. math. 166 (1994), pp. 139-213.

[28] M. Goresky, G. Harder, R. MacPherson et A. Nair, Local intersection cohomology of Baily-Borel compactifications, Compositio Math. 134 (2002), n3, pp. 243-268.

[29] M. Goresky, R. Kottwitz and R. MacPherson, Discrete series characters and the Lefschetz formula for Hecke operators, Duke Math. J. 89 (1997), pp. 477-554 and Duke Math. J. 92 (1998), no. 3, pp. 665-666.

[30] T. Hales, On the fundamental lemma for standard endoscopy: reduction to unit elements, Can. J. Math. 47 (1995), n5, pp. 974-994.

[31] M. Harris and R. Taylor, The geometry and cohomology of some simple Shimura varieties, Annals of Mathematics Studies 151, Princeton University Press (2001).

[32] A. Huber, Mixed perverse sheaves for schemes over number fields, Comp. Math. 108 (1997), pp. 107-121.

[33] R. Kottwitz, Base change for units of Hecke algebras, Compositio Math. 60 (1986), pp. 237-250.

[34] R. Kottwitz, Shimura varieties and $\lambda$-adic representations, in Automorphic forms, Shimura varieties and L-functions, Proceedings of the Ann Arbor conference, editors L. Clozel et J. Milne (1990), volume I, pp. 161-209.

[35] R. Kottwitz, Points on some Shimura varieties over finite fields, Journal of the AMS, Vol. 5, n2 (1992), pp. 373-444.

[36] R. Kottwitz, On the $\lambda$-adic representations associated to some simple Shimura varieties, Inv. Math. 108 (1992), pp. 653-665.

[37] R. Kottwitz, unpublished

[38] J.-P. Labesse, Fonctions élémentaires et lemme fondamental pour le changement de base stable, Duke Math. J. 61 (1990), 2, pp. 519-530.

[39] K.-W. Lan, Arithmetic compactifications of PEL-type Shimura varieties, Ph.D. thesis, Harvard University (2008), http://www.math.princeton.edu/ klan/articles/cptPEL-type-thesis.pdf 
[40] R. Langlands, Some contemporary problems with origins in the Jugendtraum, Proceedings of Symposia in Pure Mathematics 28 (1974), pp. 401-418.

[41] R. Langlands and D. Ramakrishnan (editors), The zeta function of Picard modular surfaces, publications du CRM (1992), Montréal.

[42] M. Larsen, Arithmetic compactification of some Shimura surfaces, in [LR], pp. 31-46.

[43] G. Laumon and B.-C. Ngo, Le lemme fondamental pour les groupes unitaires, Annals of Math. 168 (2008), no. 2, pp. 477-573.

[44] E. Looijenga, $L^{2}$-cohomology of locally symmetric varieties, Compositio Math. 67 (1988), n1, pp. 3-20.

[45] E. Looijenga et M. Rapoport, Weights in the local cohomology of a Baily-Borel compactification, Proceedings of Symposia in Pure Mathematics 53 (1991), pp. 223260.

[46] J. S. Milne, The action of an automorphism of $\mathbb{C}$ on a Shimura variety and its special points, Progr. Math., 35, pp. 239-265, Birkhäuser, Boston, 1983.

[47] J. S. Milne, Descent for Shimura varieties, Michigan Math. J. 46 (1999), no. 1, 203-208.

[48] S. Morel, Complexes pondérés sur les compactifications de Baily-Borel. Le cas des variétés de Siegel, Journal of the AMS 21 (2008), no. 1, pp. 23-61.

[49] S. Morel, On the cohomology of certain non-compact Shimura varieties, Annals of Mathematics Studies 173, Princeton University Press (2010).

[50] S. Morel, Note sur les polynômes de Kazhdan-Lusztig, to appear in Mathematische Zeitschrift.

[51] B. Moonen, Models of Shimura varieties in mixed characteristics, Galois representations in arithmetic algebraic geometry (Durham, 1996), 267-350, London Math. Soc. Lecture Note Ser., 254, Cambridge Univ. Press, Cambridge, 1998.

[52] A. Nair, Weighted cohomology of arithmetic groups Ann. of Math. (2) 150 (1999), no. 1, pp. 1-31.

[53] B. C. Ngo, Le lemme fondamental pour les algèbres de Lie, submitted, arXiv:0801.0446

[54] I. Piatetski-Shapiro, Classical and adelic automorphic forms. An introduction, in Automorphic forms, representations, and L-functions (Proc. Symposia in Pure Math., volume 33, 1977), part 1, pp. 185-188.

[55] R. Pink, Arithmetical compactification of mixed Shimura varieties, dissertation, Bonner Mathematische Schriften 209 (1989).

[56] R. Pink, On $\ell$-adic sheaves on Shimura varieties and their higher direct images in the Baily-Borel compactification, Math. Ann. 292 (1992), pp. 197-240.

[57] R. Pink, On the calculation of local terms in the Lefschetz-Verdier trace formula and its application to a conjecture of Deligne, Annals of Math., 135 (1992), pp. 483-525.

[58] M. Rapoport, On the shape of the contribution of a fixed point on the boundary : The case of $\mathbb{Q}$-rank one, in $[\mathrm{LR}], \mathrm{p} 479-488$, with an appendix by L. Saper and M. Stern, pp. 489-491.

[59] M. Saito, On the derived category of mixed Hodge modules, Proc. Japan Acad. Ser. A Math. Sci. 62 (1986), no. 9, pp. 364-366. 
[60] M. Saito, On the Formalism of Mixed Sheaves, preprint RIMS n784 (1991), http://arxiv.org/abs/math/0611597

[61] L. Saper et M. Stern, $L^{2}$-cohomology of arithmetic varieties, Annals of Math. 132 (1990), n1, pp. 1-69.

[62] S. W. Shin, Galois representations arising from some compact Shimura varieties, to appear in Annals of Math.

[63] Y. Varshavsky, A proof of a generalization of Deligne's conjecture, Electron. Res. Announc. Amer. Math. Soc. 11 (2005), pp. 78-88.

[64] J.-L.Waldspurger, Le lemme fondamental implique le transfert, Comp. Math. 105 (1997), n ${ }^{\circ}$, pp. 153-236.

[65] J.-L.Waldspurger, Endoscopie et changement de caractéristique, J. Inst. Math. Jussieu 5 (2006), n 3 , pp. 423-525.

[66] J.-L.Waldspurger, L'endoscopie tordue n'est pas si tordue, Mem. Amer. Math. Soc. 194 (2008), no. 908.

[67] S. Zucker, $L^{2}$ cohomology of warped products and arithmetic groups, Invent. Math. 70 (1982/83), no. 2, pp. 169-218.

Department of Mathematics, Harvard University, One Oxford Street, Cambridge, MA 02138, USA

E-mail: morel@math.harvard.edu 